Poster \#1

\section{Directed Evolution of Vitreoscilla Hemoglobin (VHb) to support the Growth of Escherichia coli under Microaerobic Conditions}

\author{
Charlotte I. J. Andersson², Pauli T. Kallio', Niklas Holmberg', \\ James E. Bailey¹ \& Leif Bülow² \\ 1. Institute of Biotechnology, ETH-Zürich, Zürich, Switzerland \\ 2. Department of Pure and Applied Biochemistry, Center for Chemistry and \\ Chemical Engineering, Lund University, Lund, Sweden
}

There is a strict aerobic bacterium, Vitreoscilla stercoraria, living in stagnant ponds and decaying vegetable matter that produces a hemoglobin $(\mathrm{VHb})$ to sustain these oxygen-limiting conditions. The gene coding for this protein has been cloned and expressed in a variety of prokaryotic organisms, but also transferred in a few eukaryotic hosts leading to improved growth, increased production of recombinant proteins and enhanced yields of secondary metabolites. To further investigate these properties a randomly mutated expression library in $E$. coli based on the Vitreoscilla hemoglobin gene, vhb, was created by error-prone PCR. Several mutants with altered phenotype were obtained. Four mutants were able to reach $22-155 \%$ higher final cell densities in microaerobic fed-batch cultivations than an $E$. coli clone expressing native $\mathrm{VHb}$. The expression levels of mutated $\mathrm{VHb}$ proteins were similar to the amounts produced by the controls expressing native $\mathrm{VHb}$. The clones also synthesized functional $\mathrm{VHb}$, as their CO-binding absorbance spectra indicated absorption characteristics analogous to native $\mathrm{VHb}$ having an absorption maximum at $419 \mathrm{~nm}$ and a minimum at $437 \mathrm{~nm}$. This indicates that the amino acid mutations within the vhb gene are able to slightly change the structure of $\mathrm{VHb}$. However, the change of structure of mutant VHbs was able to improve the growth of $E$. coli under microaerobic conditions. By-product formation of these mutants was also decreased, for acetate to approximately $18-40 \%$ and for ethanol up to $60 \%$ of final concentration in cultures expressing native $\mathrm{VHb}$. These findings indicate that there is a correlation between a decreased by-product formation and an improved respiratory efficiency in the $E$. coli cell caused by mutations within the vhb gene. Furthermore, the production of a recombinant marker protein $\beta$-lactamase was increased up to five times for mutants compared to the strain producing native $\mathrm{VHb}$. These results show that an E. coli cell expressing mutant $\mathrm{VHb}$ is able to direct more carbon towards cell growth and recombinant protein production relative to a control cell. Our intention is now to study the effects of the most interesting mutant VHbs in higher eukaryotes.
Poster \#2

\section{Dinitroaniline Herbicide Resistant Transgenic Plants}

\author{
Richard G. Anthony, Stefanie Reichelt \& Patrick J. Hussey
}

School of Biological Sciences, Royal Holloway, University of London, Egham, Surrey TW20 OEX, UK

The dinitroaniline herbicides are used for the selective control of weeds in arable crops. We have used an $a / b$ tubulin chimeric gene construct containing a mutated a tubulin coding sequence which is known to confer dinitroaniline herbicide resistance in the invasive weed, goosegrass, to transform and regenerate tobacco plants. The results show that expression of this construct confers a dinitroaniline resistant phenotype on the tobacco and that this is stably inherited. In all the transformed lines, transgene $\mathrm{a}$ and $\mathrm{b}$ tubulins increase the overall cytoplasmic pool of tubulin by approximately 1.5 fold, whilst endogenous a and $b$ tubulin synthesis is repressed (in some tissues by up to $45 \%$ ). Overexpression of transgene $\mathrm{a}$ and $\mathrm{b}$ tubulin occurs in every plant tissue analysed and they are the principal tubulin isotypes in these tissues (comprising approximately $66 \%$ of the total tubulin content). Immunolocalisation studies reveal that the transgene $\mathrm{a}$ and $\mathrm{b}$ tubulins are incorporated into all four microtubule arrays indicating that they are functional. The fact that the transgene tubulins are the principle components of the $\mathrm{a} / \mathrm{b}$ tubulin pools implies that these can perform the majority, if not all the roles, of microtubules in both juvenile and adult tobacco plants.

Poster \#3

\section{In Search of Transgenic Food Safety}

\author{
Luc De Bry
}

Research Department, General Biscuits Belgium, Waverstraat 1, B-2860 SintKatelijne Waver, Belgium

Although very little known in plant and agricultural biotechnology circles, in our constant search for food safety ${ }^{1}$, the Maillard reaction, a top ten classic of food-physical chemistry literature, may be a gateway for alleviating consumer instinctive anxieties about novel food products from transgenic crops. A proteagineous crop, an oleagineous crop and an amylaceous 